\title{
Omega-3 supplementation in the treatment of polycystic ovary syndrome (PCOS) - a review of clinical trials and cohort
}

\author{
Vitoria Melo ${ }^{1}$, Thomas Silva ${ }^{1}$, Thaissa Silva ${ }^{1}$, Juliana Freitas ${ }^{1}$, Joselita Sacramento ${ }^{1}$, \\ Mirian VazQuez ${ }^{1}$, Edilene Araujo ${ }^{1,2,3}$
}

${ }^{1}$ Life Sciences Department, University of the State of Bahia (UNEB), Bahia, Brazil; ${ }^{2}$ Life Sciences Departmen, Nucleus of Research and Extension in Nutritional Genomics and Metabolic Dysfunctions (GENUT), University of the State of Bahia (UNEB), Salvador, Brazil; ${ }^{3}$ Post-graduation Program Interactive Process of Organs and Systems, Health Sciences Institute/UFBA, Salvador, Brazil

E-mail:emaraujo@uneb.br

\begin{abstract}
Polycystic ovary syndrome (PCOS) is the most common endocrine disorder in women associated with cardiovascular disease and obesity. The possible benefits of omega-3 supplementation in this syndrome have been discussed much. This study is aimed to verify, based on the scientific data published, if there are any benefits in the omega-3 supplementation in the treatment of PCOS and to indicate its possible dosages for the treatment of polycystic ovary. The work consists of a systematic review of clinical trials and cohort of the MEDLINE/PubMed database from 2009 to October 2019. All studies that analyzed the omega-3 supplementation in women with PCOS were included. Cross-sectional studies, review articles, systematic reviews, meta-analysis, duplicates, studies in animals or cell culture, studies with omega-3 supplementation via food or associated with other supplementations were not included, except those involving vitamin E. In total, 21 articles were selected. Despite the heterogeneity of the studies selected, indirect benefits were observed mainly regarding the glycemic profile, such as insulin resistance reduction, lipid profile modulation (i.e. decrease in total cholesterol, triglycerides, and elevation of high-density lipoprotein), and the regulation of the androgenic profile. As for the anthropometric profile, the studies were scarce and most of them had no significant meaning. Regarding the antioxidant profile and inflammatory biomarkers, the findings differ among studies, but promising results were observed with different doses over 12 weeks of use, such as C-reactive protein (CRP) reduction. Thus, omega-3 fatty acids promote indirect benefits in the treating of women with PCOS. However, to reveal well-defined standards for dosage and supplementation time, further studies are needed.
\end{abstract}

Key words: polycystic ovary syndrome, fatty acids, omega-3, fish oil, linseed oil

Polycystic ovary syndrome (PCOS) is characterized by clinical, endocrine, and metabolic disorders, including hyperandrogenism associated with the insulin resistance (de Sousa et al. 2013). It is the most common endocrine disorder in women, whereas about $6-10 \%$ of women in reproductive age has this condition (Baptiste et al. 2010). However, although such high prevalence of the syndrome has been observed, the etiology of PCOS is still not completely known. However, it is evident that insulin resistance may play an important role due to insulin stimulation to produce ovarian androgens (Silva et al. 2006; Goodarzi et al. 2011). The traditional treatment of PCOS is usually based on oral contraceptives. However, these drugs may promote several side effects, including blood clots formations, headache, weight gain, breast cancer, and depletion of different micronutrients (folic acid and vitamins B2, B6,

Corresponding author: Dr. Edilene Araujo, Universidade do Estado da Bahia, Departamento de Ciencias da Vida, predio 1. Rua Silveira Martins, 2555, Cabula, Salvador, Bahia, Brasil, 41150-000; phone: +55 71 3117-2289; e-mail: emaraujo@uneb.br. 
B12, C and E) (Palmery et al. 2013; Stegeman et al. 2013; Mohammad-Alizadeh-Charandabi et al. 2015; $\mathrm{Li}$ et al. 2017). Given this context, it is important to understand alternative therapies to promote improvements in the health status and quality of the life. Weight loss, caloric restriction, and intake of inadequate nutrients have contributed to the significant reduction of the symptoms associated with PCOS (Moran et al. 2009; Nybacka et al. 2011) since the syndrome has inflammatory characteristics that change the glycemic and lipid metabolism (Goodarzi et al. 2011). Due to this inflammatory feature of PCOS, the consumption of the omega- 3 fatty acids may be a promising nutritional strategy to treat the disease regarding to its anti-atherogenic and antiinflammatory effects. In addition, the omega- 3 fatty acids might have beneficial effects on the glycemic control and lipid profile (Becic and Studenik 2018; Thota et al. 2018). Therefore, this study is aimed to verify, based on the scientific literature data, if there are benefits in the omega-3 supplementation in the treatment of PCOS as well as indicates possible dosages for the treating of this syndrome.

\section{Materials and methods}

This work is a systematic review of selected articles, based on PRISMA (Preferred Reporting
Items for Systematic Reviews and Meta-Analyses) recommendation, to evaluate the possible effects of the omega-3 supplementation for PCOS cases. Scientific literature was researched to identify the relevant studies to this review by using the MEDLINE/PubMed database until October 2019. Inclusion criteria were that all articles published in the last 10 years (2009-2019) contained full text and the following characteristics: clinical trials, randomized/cohort, in which oral animal and/or vegetal omega-3 supplementation has been analyzed and applied to women with PCOS. Those studies were included, in which omega-3 supplementation was associated with vitamin $\mathrm{E}$ as the most common commercial association. Cross-sectional studies, review articles, duplicate publications, studies in animals or cell culture studies that analyzed omega-3 supplementation via food or associated with another supplementation, except vitamin E, were not included. In the search for publications, the logical AND operator was used to combine the term Ovary Polycystic Syndrome with the following terms: omega 3; fish oil; linseed oil; alpha linolenic acid; linolenic acid; polyunsaturated fatty acids; eicosapentaenoic acid, and docosahexaenoic acid. Three reviewers independently carried out the selection and analysis of articles by using the predefined eligibility criteria (Figure 1).

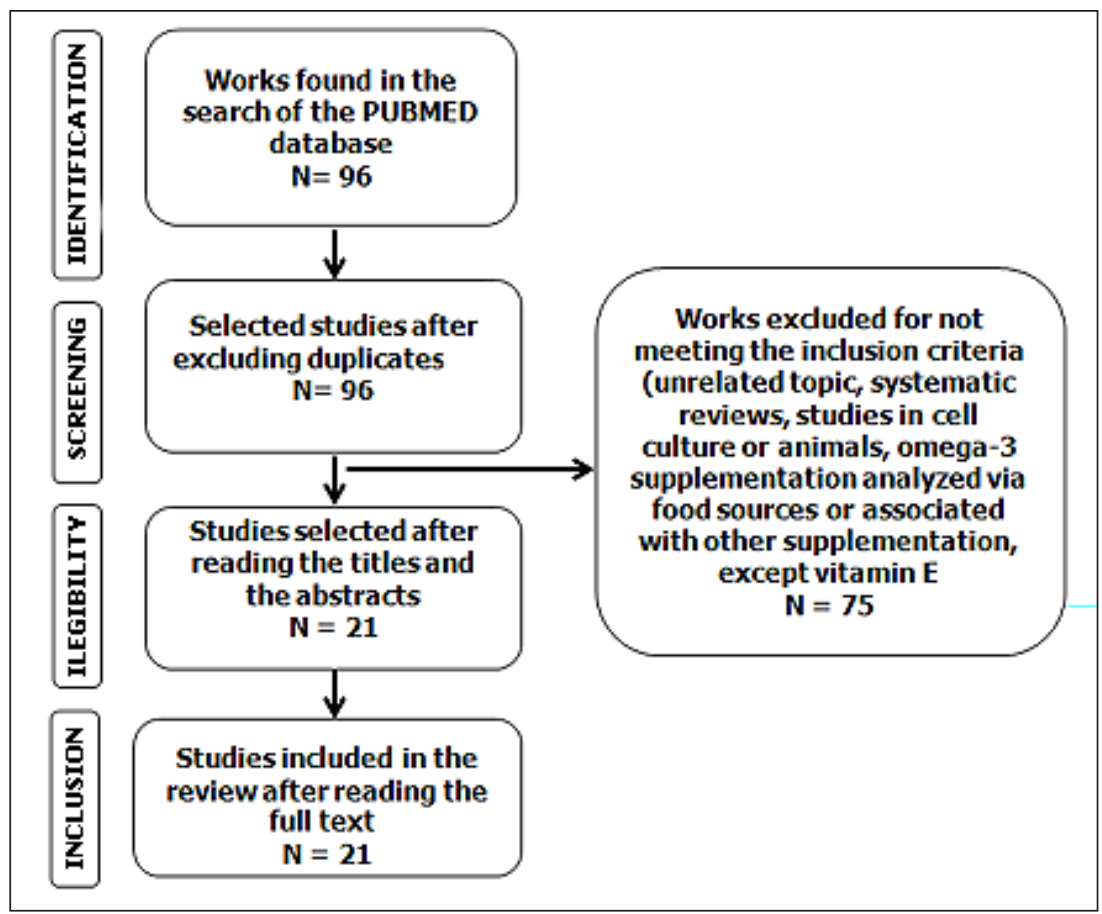

Figure 1. Flowchart of the systematic search for articles. 


\section{Results}

Altogether, 96 articles were obtained without duplicates. After reading the titles and abstracts, 21 studies were selected. The main reason for excluding the articles was the non-adequacy to the proposed theme or studies done on animals or in vitro conditions. After the reading completing, 21 articles remained in the study, which met the criteria established (Figure 1). The average time for clinical trials in this review endured from 6 to 12 weeks (only two articles lasted 6 months) and an average of 63.7 women participated in the study. The age of these women ranged between 18 and 45 years. Out of the 21 clinical trials, 12 used fish oil as a source of omega- 3 supplementation; 2 used omega-3 fat associated with vitamin E; 2 used linseed oil; 3 used linseed oil associated with vitamin E; and 2 used fish oil and linseed oil in the same study. Parameters associated with PCOS were analyzed, such as lipid, glycemic, hormonal profiles, inflammation and oxidative stress biomarkers, and anthropometric parameters. The results are described in the Table 1.

\section{Discussion}

According to the studies analyzed, supplementation with animal/vegetable origin the omega-3 fatty acids might have indirect beneficial effects on PCOS via the significant improvement of parameters of the lipid, glycemic profile, in addition to some effects on the hormonal profile and antioxidant and inflammatory biomarkers. However, controversial results were found, which might be related to different dosages, supplementation time, and the sample size. It is noteworthy that the studies included in this review simultaneously analyzed several aspects related to PCOS. For a better understanding, several aspects will be discussed below in separate topics.

Effect on lipid profile and hepatic fat. The omega-3 consumption is related to the improvement of the lipid profile and reduction of cardiovascular risk since fatty acids are natural ligands of the metabolic nuclear receptors, such as PPARs (peroxisome proliferator-activated receptors), and SREBP-1 (sterol regulating element binding protein 1). Activation of these omega-3 receptors can inhibit coding of proteins that stimulate lipid synthesis and stimulate genes that increase lipid oxidation in liver and muscle (Cussons et al. 2009; Mohammadi et al. 2012; Rafraf et al. 2012). In addition, the omega-3 supplementation seems to play an important role in the activation of AMPK (AMP-activated protein kinase), a sensor of cellular energy status that regulates the balance between lipid oxidation and lipogenesis. Ingestion of the omega- 3 supplementation also increases the activity of the low-density lipoprotein (LDL) receptor in the liver, which reduces LDL-cholesterol synthesis and thus, increases the LDL-cholesterol catabolism rate (Cussons et al. 2009; Mohammadi et al. 2012; Rafraf et al. 2012). This effect has been observed in women with high omega-3 plasma levels, which resulted in a significant improvement in the lipid profile for triglycerides and triglyceride ratio: highdensity lipoprotein (HDL) (Phelan et al. 2011; Vargas et al. 2011; Karakas et al. 2016).

In addition, it has been noted that the use of fish and linseed oils provides a significant reduction in serum triglycerides, without changing the total cholesterol (TC) levels, HDL or apoprotein B (Vargas et al. 2011). However, the omega-3 consumption from linseed oil associated with vitamin E significantly decreases serum triglycerides, very low-density lipoproteins (VLDL), LDL, and TC, as well as reduces the lipoprotein A (Lp [a]) and oxidized LDL (Ox-LDL) mRNA in peripheral blood mononuclear cells (Rahmani et al. 2016). Reduction of TC indices and their fractions were noted after supplementation with fish oil, in addition to a significant reduction in LDL and an increase in HDL (Mohammadi et al. 2012; Khani et al, 2017; Mejia-Montilla et al. 2018; Yang et al. 2018).

The effects of the omega-3 oils on triglyceride levels, HDL, LDL, and TC seem to be contradictory due to the heterogeneity of the studies (Arentz et al. 2017). It is noticeable that the dosages and the supplementation time were very different among studies, which makes standardization difficult. From the 12 clinical trials that analyzed lipid profile, 11 found positive results regarding the modulation of levels. The study without significant results analyzed a dosage of 800 $\mathrm{mg}$ of omega-3 supplementation from fish oil (Amini et al. 2018). The reduction in triglycerides was mostly found in the studies analyzed (Mohammad-Alizadeh-Charandabi et al. 2015). As for supplementation, the procedures with best results in the treatment of lipid profile women with PCOS were as follows: $1200 \mathrm{mg}$ /day of omega-3 supplementation from fish oil (eicosapentaenoic acid, EPA+docosahexaenoic acid, DHA), for 8 weeks (2 studies) (Mohammadi et al. 2012; Rafraf et al. 2012) and $600 \mathrm{mg} /$ day (EPA+DHA) from fish oil for 6 months (1 study) (Oner and Muderris 2013). For linseed-oil-based omega-3 supplementation, the most effective results were $800 \mathrm{mg}$ alpha-linolenic acid (ALA)/day for 12 weeks (1 study) (Mirmasoumi et al. 2018) and $400 \mathrm{mg}$ 


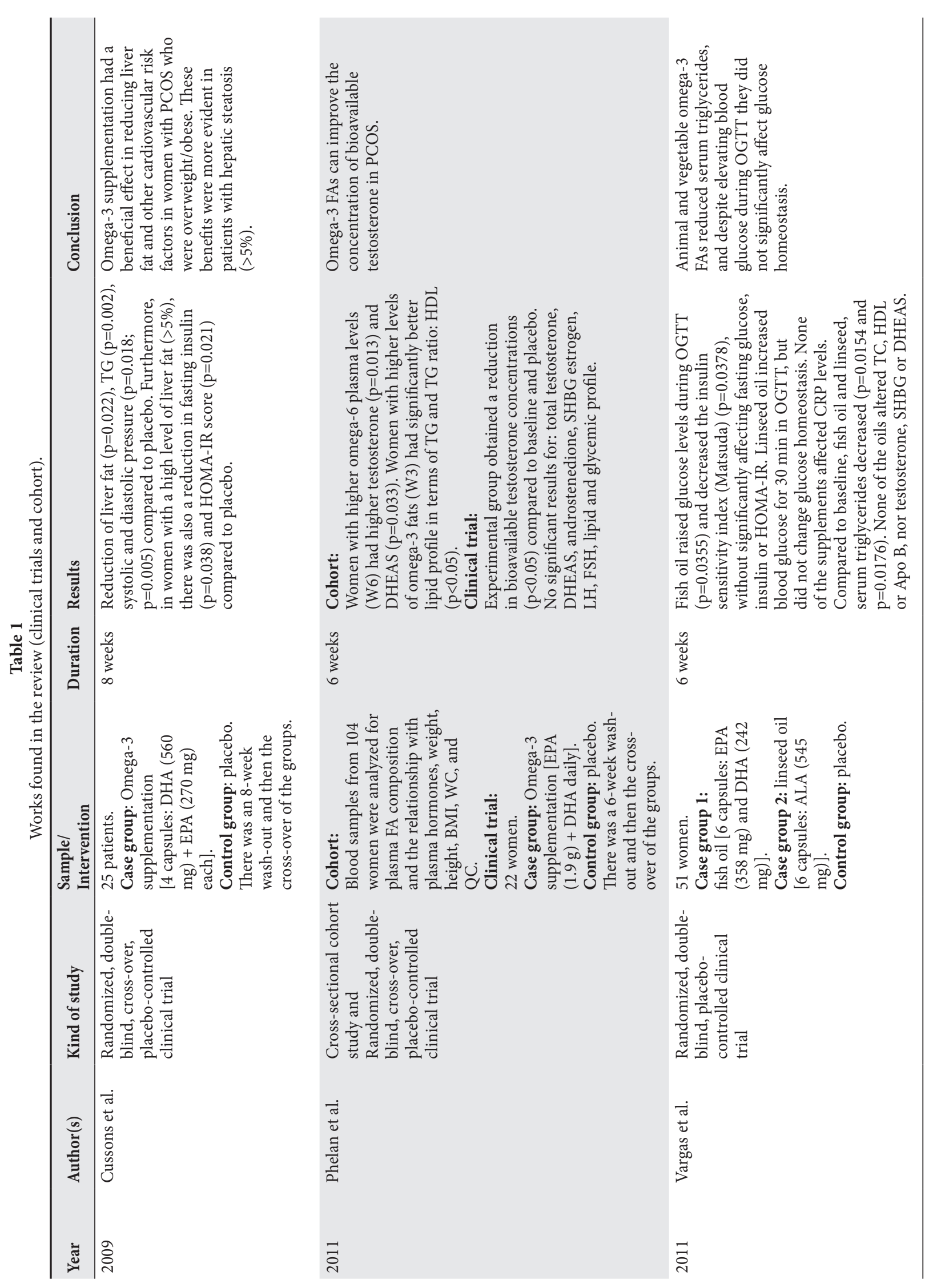




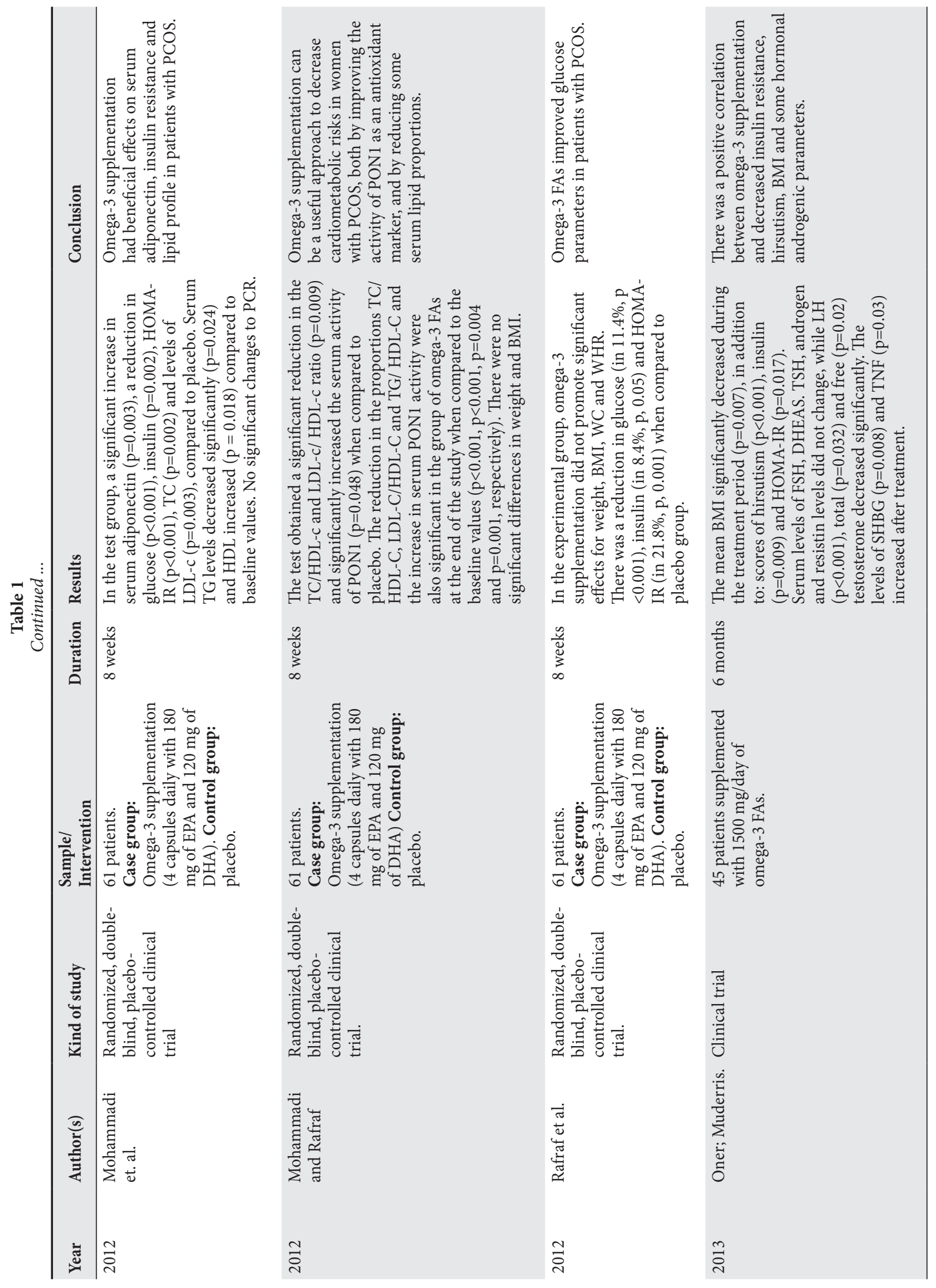




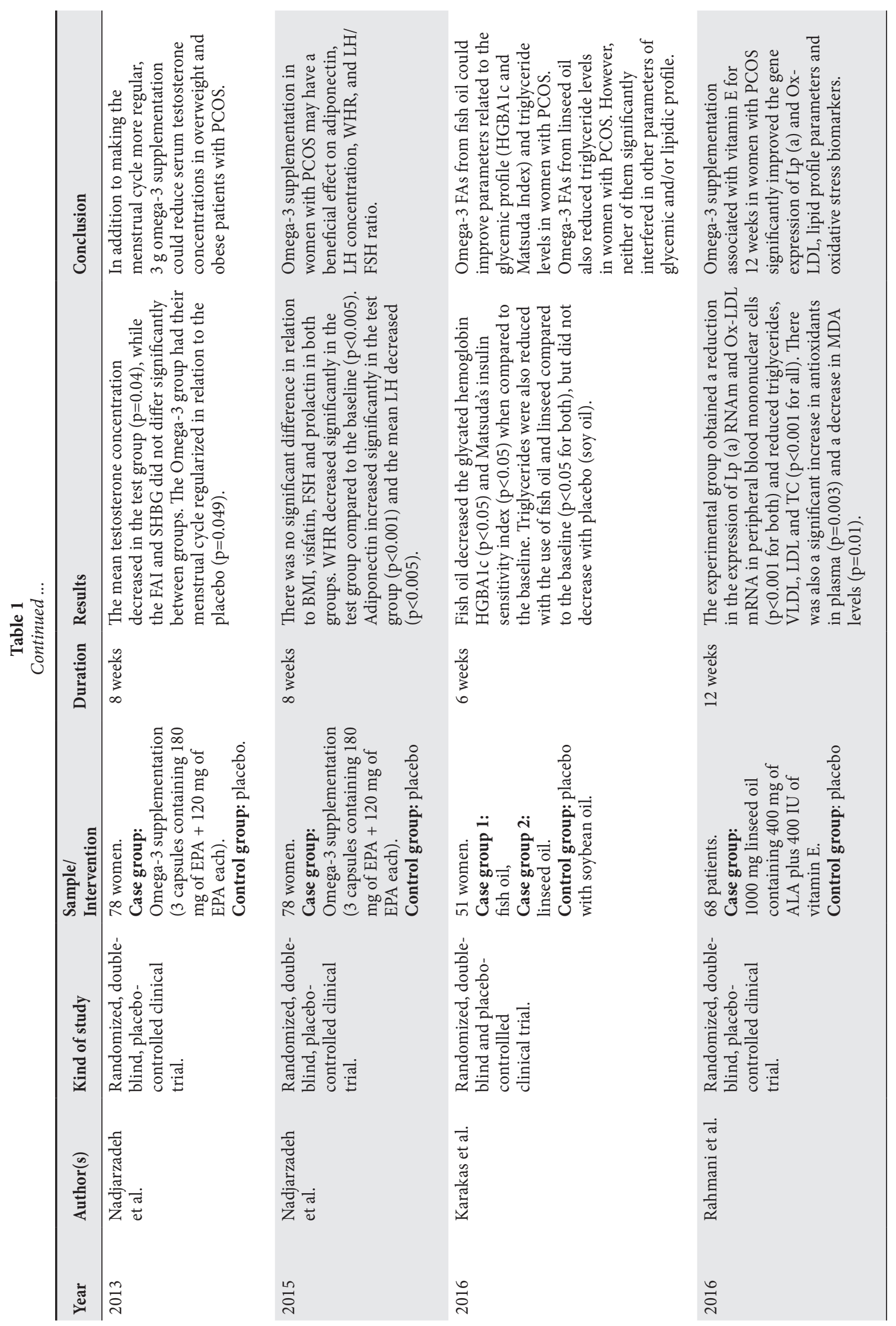




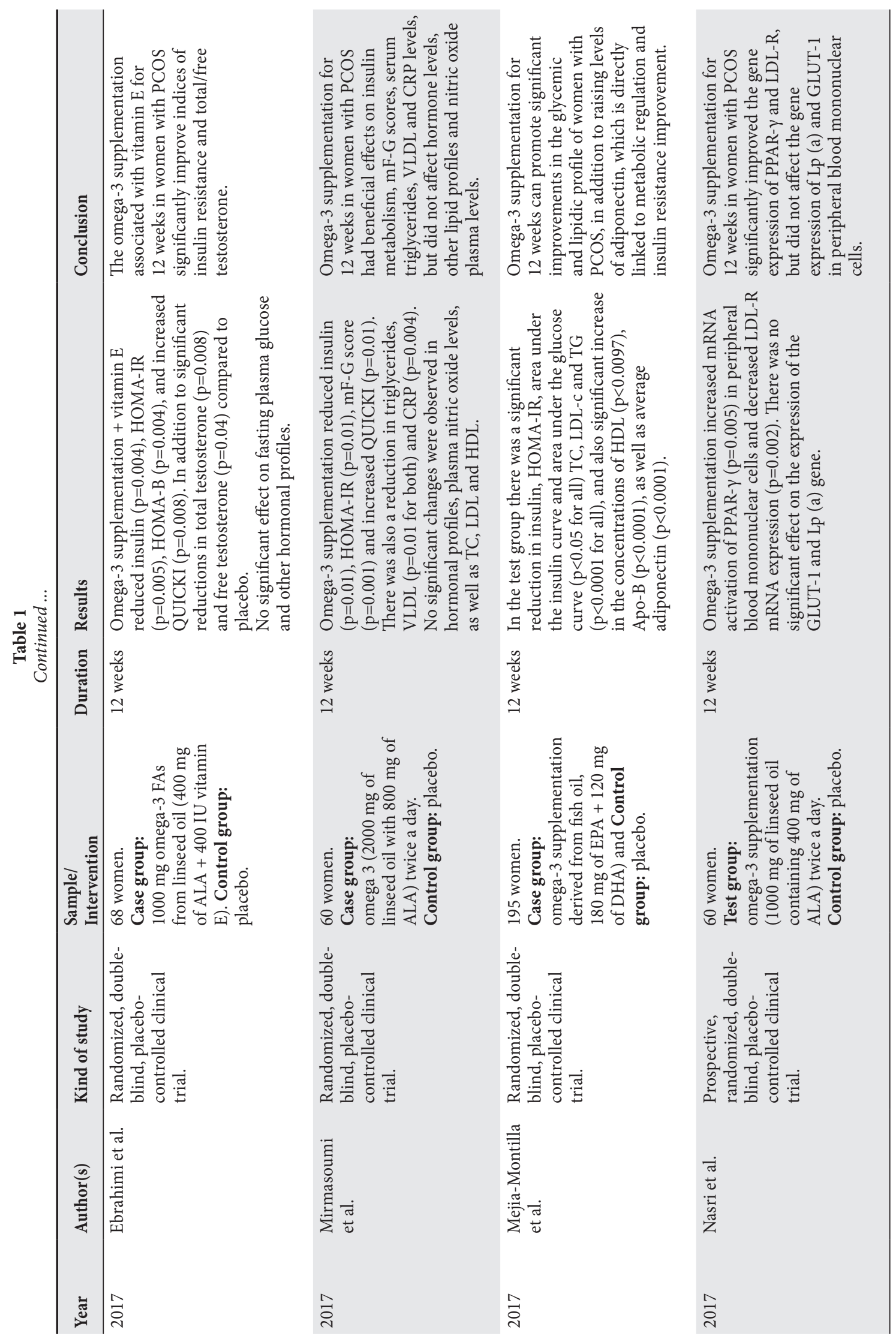




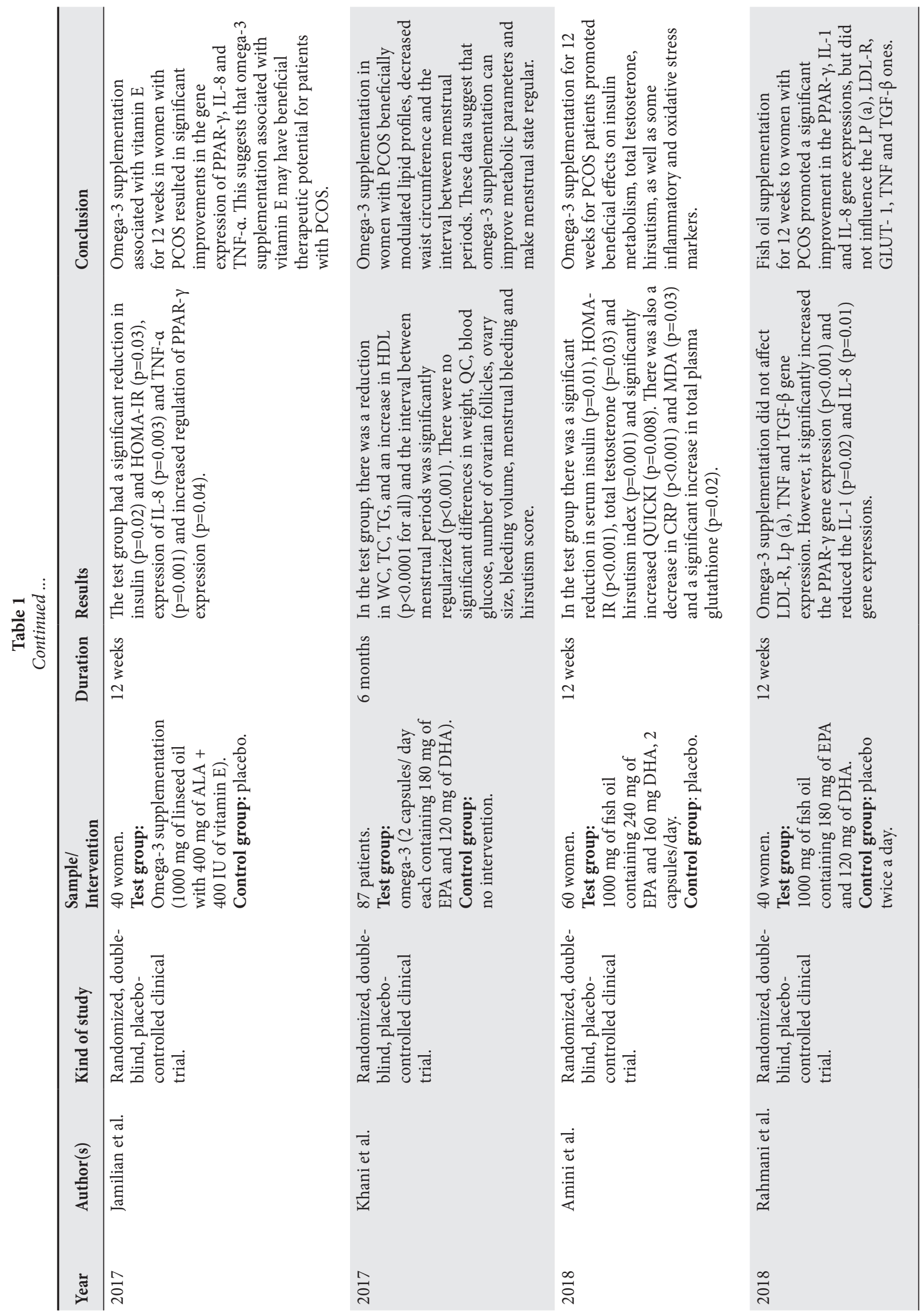




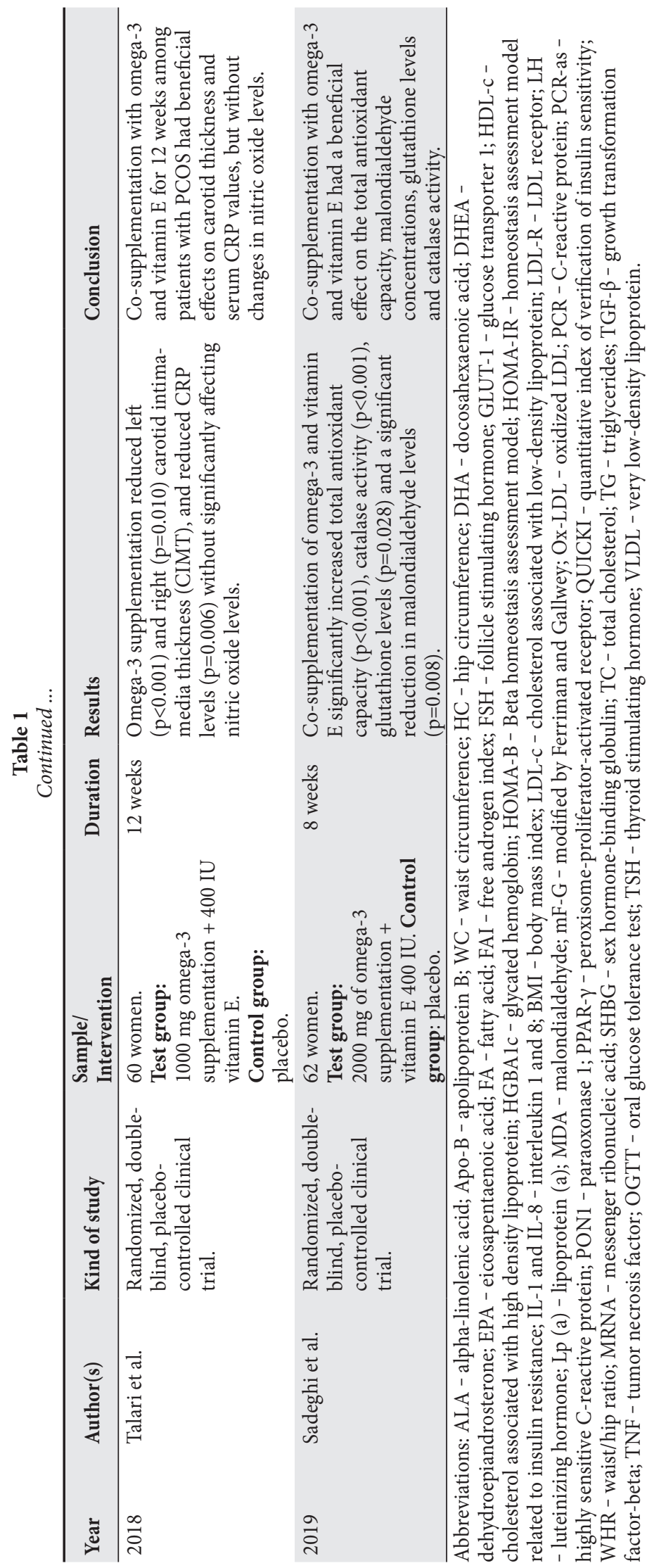

ALA+400 IU vitamin E/day for 12 weeks (1 study) (Rahmani et al. 2016). As for hepatic steatosis, the only data found for $3.32 \mathrm{~g}$ of $\mathrm{EPA}+\mathrm{DHA} / \mathrm{day}$ for 8 weeks. In this case, a reduction of $>5 \%$ of hepatic fat reduction was observed $(\mathrm{p}=0.022)$ (Cussons et al. 2009).

Effect on the steroidogenic profile. Hyperandrogenism is a hallmark of PCOS related to hyperinsulinemia, as this factor in the syndrome is associated with a direct increase in the production of androgens by the ovary and a decrease in the levels of sex hormone-binding globulin (SHBG). SHBG is a protein that binds to testosterone, reducing its action potential. Therefore, after the reduction of SHBG caused by hyperinsulinemia, there is a massive increase of free testosterone. In addition, patients with PCOS have hypersecretion of the luteinizing hormone (LH), which further increases the production of androgens and causes disturbance of the menstrual cycle, infertility, hirsutism, acne, and alopecia (Hajishafiee et al. 2016; Yao et al. 2017; Amini et al. 2018).

Clinical trials have demonstrated a possible anti-androgenic function of omega-3 fatty acids in PCOS. Although the mechanisms have not been well established, insulin reduction is an important factor of these fatty acids (Oner and Muderris 2013; Ebrahimi et al. 2017; Khani et al. 2017). This relationship was well demonstrated in a study that observed the omega-6/omega-3 ratio in the blood plasma of women with PCOS and concluded that the higher the omega-6/omega-3 ratio in the plasma, the higher levels of testosterone and dehydroepiandrosterone (DHEA-S) (Phelan et al. 2011). The omega-3 supplementation of 1.9 $\mathrm{g}(\mathrm{EPA}+\mathrm{DHA})$ for 6 weeks can reduce free testosterone levels without interfering with the total testosterone, DHEA-S, androstenedione, SHBG, estrogen, LH or folliclestimulating hormone (FSH). However, ALA supplementation of $400 \mathrm{mg}$ ALA+400 IU of vitamin $\mathrm{E}$ for 12 weeks promoted significant reductions not only in the free testosterone, but also in the total plasma testosterone and decreased hirsutism index when compared to the placebo group (Ebrahimi et al. 2017; Amini et al. 2018). Similarly, the omega-3 supplementation of $800 \mathrm{mg}$ for 12 weeks 
or $1500 \mathrm{mg}$ for a period of more than 6 months significantly reduced several steroidogenic parameters, such as serum levels of the total testosterone, free testosterone, LH, androgens, and hirsutism score (Oner and Muderris 2013; Amini et al. 2018). Lower doses of EPA+DHA (600 mg/day) did not reduce the hirsutism index, but the menstrual cycle of women with PCOS became more regular (Khani et al. 2017). The same result was found in the fish oil omega-3 supplementation of $900 \mathrm{mg}$ since there was a reduction in the mean testosterone concentration (Nadjarzadeh et al. 2013) and the LH levels (Nadjarzadeh et al. 2015).

In contrast to these findings, supplementation with a higher dosage of $3.6 \mathrm{~g}$ of EPA+DHA for 6 weeks did not induce effects on testosterone, SHBG or DHEAS (Vargas et al. 2011). The omega-3 supplementation may have an effect in reducing the total testosterone, but it does not reduce both SHBG and DHEA-S, as the results were controversial and this supplementation is suggested for longer periods ( $>6$ weeks) to obtain the same effect (Hajishafiee et al. 2016). The effects found in the studies were widely different, as well as their doses and supplementation time. Among the 8 clinical trials analyzing steroidogenic hormonal aspects, only one did not find any benefit in this profile (Vargas et al. 2011). However, this study had the highest dosage (3.6 g of EPA+DHA) in a reduced period of time, which raises the discussion that the standard dose used (1.5 g of EPA+DHA) for long periods ( $>6$ months) may show better benefits (Oner and Muderris 2013).

Effect on the glycemic profile. As already discussed, the PCOS pathogenesis may have a direct link to insulin resistance since hyperinsulinemia promotes hyperandrogenism by stimulating androgen synthesis via teak cells and reduces SHBG and thus, produces free testosterone increasingly (Avila et al. 2014; Sadeghi et al. 2017). Studies have shown that women with PCOS have an increased risk of type 2 diabetes mellitus (DM2) in comparison to non-PCOS women, and more than $50 \%$ of patients with PCOS are insulin resistant (Unluhizarci et al. 2012; Jamil et al. 2015). Therefore, the discussion arises about the possible relationships between the omega-3 fatty acids, insulin resistance, and PCOS. Several studies have found positive results from omega-3 supplementation on glycemic parameters and adiponectin levels (Mohammadi et al. 2012; Ebrahimi et al. 2017; Amini et al. 2018; Jamilian et al. 2018). Adiponectin is a hormone that improves sensitivity of cells to insulin and has an anti-inflammatory and antiatherogenic effects. The possible beneficial effects of omega-3 supplementation on glucose parameters can be partially associated with this fatty acid potential to increase adiponectin production (Mohammadi et al. 2012). The significant increase of this hormone was noticed after 8 weeks of the omega-3 supplementation (900 mg of EPA+DHA) (Nadjarzadeh et al. 2015). However, in addition to significantly increasing levels of adiponectin, the use of $1200 \mathrm{mg}$ of EPA+DHA for the same period favored the reduction of glucose, insulin, and HOMA-IR (Mohammadi et al. 2012; Rafraf et al. 2012).

In a longer supplementation period (6 months) with $1500 \mathrm{mg}$ of $\mathrm{EPA}+\mathrm{DHA} /$ day, levels of insulin and HOMA-IR were reduced without a significant reduction in blood glucose (Cussons et al. 2009; Oner and Muderris 2013). Some studies did not find significant changes in insulin, glycemia, and HOMA-IR levels even after higher omega-3 doses (1.9 g and $3.6 \mathrm{~g}$ for 6 weeks) (Phelan et al. 2011; Vargas et al. 2011). A meta-analysis concluded that there was no significant effect of the omega-3 supplementation, when compared to placebo, on insulin resistance, and HOMA-IR in women with PCOS (Sadeghi et al. 2017). However, recent studies have shown promising results in this regard. The omega- 3 supplementation from linseed oil with vitamin E co-supplementation (400 mg ALA+400 IU vitamin E) for 12 weeks had benefits on the glycemic profile of women with PCOS, such as reduced insulin and HOMA-IR (Ebrahimi et al. 2017; Jamilian et al. 2018). Similar findings were found with the linseed oil supplementation without vitamin E (800 $\mathrm{mg}$ of ALA) (Mirmasoumi et al. 2018), fish oil supplementation (dosage not reported) (Mejia-Montilla et al. 2018), and fish oil supplementation (800 mg of EPA+DHA) (Amini et al. 2018) all lasting 12 weeks. The heterogeneity of the studies has revealed contradictory results in relation to the omega-3 supplementation in women with PCOS and changes in the glycemic profile. However, the beneficial effects are clearly evident (Arentz et al. 2017; Yang et al. 2018). According to the analyses, the omega-3 supplementation may have benefits in reducing insulin and HOMA-IR in women with PCOS. In 12 clinical trials that evaluated at least 1 parameter related to the glycemic profile, 10 found positive results mainly in the longer supplementation periods. From the 12 studies, 5 found reduced insulin and HOMA-IR within 12 weeks of supplementation, either with linseed oil (400 mg ALA+400 IU vitamin $\mathrm{E} /$ day or $800 \mathrm{mg} \mathrm{ALA} /$ day without adding vitamin E) or fish oil (1200 or $1500 \mathrm{mg} /$ day of EPA+DHA).

Effect on the anthropometric profile. Women with PCOS have a higher prevalence of obesity and metabolic syndrome since the correlation between 
obesity, insulin resistance, and PCOS pathogenesis (Oner and Muderris 2013; Silva 2013). Therefore, diets and healthy eating patterns for weight loss have been widely discussed in the treatment of this disease. A study that proposed a diet with a healthy eating pattern for women with PCOS has shown significant weight loss in these women in addition to improvements in steroidogenic parameters and the glycemic profile related to the syndrome. Thus, weight loss is related to a positive impact of PCOS treatment (Foroozanfard et al. 2017). It has been discussed much in relation to the effects of omega- 3 fatty acids on weight loss since some studies have made this association. A trial carried out with diabetic women observed a reduction in adiposity after the omega-3 supplementation (Lee et al. 2013). Although there are significant results of weight loss in rodents (Buckley and Howe 2010), studies in humans that demonstrate weight loss with the omega- 3 supplementation are still scarce. There were no significant differences in body mass index (BMI) and body weight after using $1200 \mathrm{mg}$ omega-3 supplementation (EPA+DHA) for 8 weeks in women with PCOS (Rafraf et al. 2012; Mohammadi and Rafraf 2012). However, a significant reduction in mean BMI has been observed in a group of women supplemented with omega- 3 after 6 months of treatment, with $1500 \mathrm{mg}$ of EPA+DHA (Oner and Muderris 2013). For the same period, the omega-3 supplementation of 2000 favored a reduction in waist circumference (WC) without changes in weight (Khani et al. 2017). After 8 weeks of the omega-3 supplementation of $900 \mathrm{mg}(\mathrm{EPA}+\mathrm{DHA})$, there was a significant decrease in the waist-to-hip ratio (WHR) compared to baseline, but BMI did not change significantly (Nadjarzadeh et al. 2015). Data suggest that there is an association between omega-3 fatty acids for long periods and reduction in body measurements, even though there is no strong evidence that the omega-3 supplementation has a significant effect on BMI (Yang et al. 2018).

Effect on the inflammation and oxidative stress biomarkers. PCOS is related to a chronic inflammatory process with elevated serum levels of proinflammatory markers. Pro-inflammatory cytokines, such as tumor necrosis factor (TNF) and interleukin 6 (IL-6), are important factors for the insulin resistance development (Escobar-Morreale et al. 2011; Oner and Muderris 2013). Furthermore, this syndrome is also associated with oxidative stress, in which the increased production of free radicals and reactive oxygen species (ROS) are made for the decreased levels of plasma antioxidants (Gonzalez et al. 2013). Therefore, the omega- 3 supplementation could be beneficial for women with PCOS as these fatty acids act in competition with arachidonic acid leading to the inhibition of the production of pro-inflammatory eicosanoids. These eicosanoids serve as an alternative substrate for cyclooxygenase (COX and COX2), which results in the formation of less potent products than pro-inflammatory ones (Barbalho et al. 2011).

There are evidences that omega-3 supplementation significantly increases the body's antioxidant capacity as well as reduces the levels of malondialdehyde (MDA) (Najafi et al. 2017; Ali and Rifaai 2019). However, no satisfactory results were found for C-reactive protein (CRP) levels reduction after administration of $1.2 \mathrm{~g}$ fish oil omega-3 supplementation for 8 weeks (Mohammadi et al. 2012) or $3.6 \mathrm{~g}$ of fish/ flax oil omega-3 supplementation (3.2 g) for 6 weeks (Vargas et al. 2011). A significant reduction in CRP levels was noticed with $800 \mathrm{mg}$ ALA supplementation (Mirmasoumi et al. 2018), $1000 \mathrm{mg}$ of omega-3+400 UI vitamin $\mathrm{E}$, both over a 12 -week period (Talari et al 2018) $800 \mathrm{mg}$ of EPA+DHA also decreased the levels of CRP and malondialdehyde with a significant increase in total plasma glutathione, which points out body's antioxidant efficiency (Amini et al. 2018). The increase in the serum activity of paraoxonase 1 (PON1), an antioxidant enzyme linked to HDL, has been observed after administration of $1200 \mathrm{mg}$ of EPA+DHA for 8 weeks (Mohammadi and Rafraf 2012). Other findings showed a significant increase in the levels of total plasma antioxidants and a decrease in malondialdehyde levels after using $400 \mathrm{mg}$ of ALA+400 IU vitamin E, for 12 weeks (Rahmani et al. 2016). Based on the same dosage and supplementation time, there was an increase in TNF, PPAR- $\gamma$, and IL-8 gene expressions, but without influencing the glucose transporter type 1 (GLUT-1), IL-6, and transforming growth factor $\beta$ (TGF- $\beta$ ) levels (Jamilian et al. 2018 ) and $2000 \mathrm{mg}$ of omega-3 supplementation +400 IU of vitamin E for 8 weeks significantly increased the total antioxidant capacity, catalase activity, glutathione levels, and reduced MDA levels (Sadeghi et al. 2020). The omega-3 supplementation can increase TNF levels since it reduces insulin levels, which is a hormone associated with anti-inflammatory effects. Therefore, insulin reduction could influence the TNF elevation (Oner and Muderris 2013). Fish oil supplementation for 12 weeks for women with Polycystic Ovary Syndrome also improved complementing PPAR- $\gamma$, IL-1 and IL-8 gene expression, but did not influence LP(a), LDLR, GLUT gene expression -1, TNF- $\alpha$ and TGF- $\beta$ (Rahmani et al. 2018).

In the analyzed studies, it was observed that the results are scarce and quite controversial regarding 
the effects of the omega- 3 fatty acids on inflammatory biomarkers and oxidative profile in women with PCOS. In total, 12 clinical trials evaluated at least one aspect related to these profiles, whereas only 3 of them were found with a CRP reduction. They all lasted 12 weeks of supplementation with different doses as follows: $1000 \mathrm{mg}$ of $\mathrm{EPA}+\mathrm{DHA}+400 \mathrm{IU}$ of vitamin $\mathrm{E}$ or $800 \mathrm{mg}$ of ALA or $800 \mathrm{mg}$ of EPA+DHA. In 3 cases, an improvement in the PPAR- $\gamma$ gene expression also lasting 12 weeks and different doses as follows: 400 $\mathrm{mg}$ of ALA or $400 \mathrm{mg}$ of ALA+400 IU vitamin E or $600 \mathrm{mg}$ EPA+DHA was found.

\section{Conclusions}

Despite the fact that the selected studies did not show any direct benefits of the omega- 3 supplementation in PCOS, this fatty acid clearly promotes indirect benefits by improving the metabolic profile associated with the disease. However, due to the great heterogeneity of the studies, it became difficult to standardize the dosages and the time of use. However, more efficient results could be noticed in the lipid and glycemic profiles with dosages between 1200 to $1500 \mathrm{mg}$ of the fish-oil EPA+DHA/day, 800 $\mathrm{mg}$ of ALA/day or $400 \mathrm{mg}$ of ALA+400 IU of vitamin $\mathrm{E}$ from oil linseed during the same time between 8 and 12 weeks. As for the androgenic profile, the dosage of 1200 to $1500 \mathrm{mg}$ of EPA+DHA seems to be necessary for a longer period, i.e. 6 months of use. As for the anthropometric profile, the studies analyzed in women with PCOS are scarce and incongruous regarding the benefits from weight loss and/or body fat. For the antioxidant profile and biomarkers of the inflammatory process, positive results were observed for the reduction of CRP and regulation of PPAR- $\gamma$ gene expression with different doses above 12 weeks of use. Since the results are promising, further studies should be performed with better-defined standards regarding the dosage and the supplementation time.

\section{References}

Ali FF, Rifaai RA. Preventive effect of omega-3 fatty acids in a rat model of stress-induced liver injury. J Cell Physiol 234, 11960-11968, 2019.

Amini M, Bahmani F, Foroozanfard F, Vahedpoor Z, Ghaderi A, Taghizadeh M, Karbassizadeh H, Asemi Z. The effects of fish oil omega-3 fatty acid supplementation on mental health parameters and metabolic status of patients with polycystic ovary syndrome: a randomized, double-blind, placebo-controlled trial. J Psychosom Obstet Gynaecol 1-9, 2018.

Arentz S, Smith CA, Abbott J, Bensoussan A. Nutritional supplements and herbal medicines for women with polycystic ovary syndrome; a systematic review and meta-analysis. BMC Complement Altern Med 17, 500, 2017.

Avila MAP, Bruno RV, Barbosa FC, Andrade FC, Silva ACO, Nardi AE. Polycystic ovary syndrome: implications of metabolic dysfunction. Rev Col Bras Cirur 41, 106-110, 2014.

Baptiste CG, Batistta MC, Trottier A, Baillargeon JP. Insulin and hyperandrogenism in women with polycystic ovary syndrome. J Steroid Biochem Mol Biol 122, 42-52, 2010.

Barbalho SM, Bechara MD, Quesada KR, Goulart RA. Role of omega-3 fatty acids in the resolution of inflammatory processes. Medicina (Online) 44, 234-240, 2011.

Becic T, Studenik C. Effects of omega-3 Supplementation on adipocytokines in prediabetes and type 2 diabetes mellitus: systematic review and meta-analysis of randomized controlled trials. Diabetes Metab J 42, 101-116, 2018.

Buckley JD, Howe PRC. Long-chain omega-3 polyunsaturated fatty acids may be beneficial for reducing obesity - a review Nutrients 2, 1212-1230, 2010.

Cussons AJ, Watts GF, Mori TA, Stuckey BGA. Omega-3 fatty acid supplementation decreases liver fat content in polycystic ovary syndrome: a randomized controlled trial employing proton magnetic resonance spectroscopy. J Clin Endocrinol Metab 94, 3842-3848, 2009.

de Sousa RML, Chen MBC, da Silva DSM, Dutra MB, Navarro PAAS, Neto JAF, Brito LMO. Metabolic profile in women of different body mass indices with polycystic ovary syndrome. Rev Bras Ginecol Obstet 35, 413-420, 2013.

Ebrahimi FA, Samimi M, Foroozanfard F, Jamilian M, Akbari H, Rahmani E Ahmadi S, Taghizadeh M, Memarzadeh MR, Asemi Z. The effects of Omega-3 fatty acids and vitamin E co-supplementation on indices of insulin resistance and hormonal parameters in patients with polycystic ovary syndrome: a randomized, doubleblind, Placebo-Controlled Trial. Exp Clin Endocrinol Diab 125, 353-359, 2017. 
Escobar-Morreale HF, Luque-Ramirez M, Gonzalez F. Circulating inflammatory markers in polycystic ovary syndrome: a systematic review and metaanalysis. Fertil Steril 95, 048-1058, 2011.

Foroozanfard F, Rafiei H, Samimi M, Gilasi HR, Gorjizadeh R, Heidar Z, Asemi Z. The effects of dietary approaches to stop hypertension diet on weight loss, anti-Müllerian hormone and metabolic profiles in women with polycystic ovary syndrome: A randomized clinical trial. Clin Endocrinol 87, 51-58, 2017.

Gonzalez F, Sia CL, Shepard MK, Rote NS, Minium J. Hyperglycemia-induced oxidative stress is independent of excess abdominal adiposity in normal-weight women with polycystic ovary syndrome. Human Repr 27, 3560-3568, 2013.

Goodarzi MO, Dumesic DA, Chazenbalk G, Azziz R. Polycystic ovary syndrome: etiology, pathogenesis and diagnosis. Nat Rev Endocrinol 7, 219-231, 2011.

Hajishafiee M, Askari G, Iranj B, Ghiasvand R, Bellissimo N, Totosy de Zepetnek J and Salehi-Abargouei A. The effect of n-3 polyunsaturated fatty acid supplementation on androgen status in patients with polycystic ovary syndrome: A systematic review and meta-analysis of clinical trials. Horm Met Res 48, 281-289, 2016.

Jamil AS, Alalaf SK, Al-Tawil NG, Al-Shawaf T. A case-control observational study of insulin resistance and metabolic syndrome among the four phenotypes of polycystic ovary syndrome based on Rotterdam criteria. Repr Health 12, 7, 2015.

Jamilian M, Shojaei A, Samimi M, Ebrahimi FA, Aghadavod E, Karamali M, Taghizadeh M, Jamilian H, Alaeinasab S, Jafarnejad S, Asemi Z. The effects of omega-3 and vitamin E co-supplementation on parameters of mental health and gene expression related to insulin and inflammation in subjects with polycystic ovary syndrome. J Affect Disord 229, 41-47, 2018.

Karakas SE, Perroud B, Kind T, Palazoglu M, Fiehn O. Changes in plasma metabolites and glucose homeostasis during omega-3 polyunsaturated fatty acid supplementation in women with polycystic ovary syndrome. BBA Clin 5, 179-185, 2016.

Khani B, Mardanian F, Fesharaki SJ. Omega-3 supplementation effects on polycystic ovary syndrome symptoms and metabolic syndrome. J Res Med Sci 22, 64, 2017.

Lee MW, Park JK, Hong JW, et al. Beneficial effects of omega-3 fatty acids on low density lipoprotein particle size in patients with type 2 diabetes already under statin therapy. Diab Metab J 37, 207-211, 2013.

Li L, Zhong Y, Zhang H, Yu H, Huang Y, Li Z, Chen G, Hua X. Association between oral contraceptive use as a risk factor and triple-negative breast cancer: A systematic review and meta-analysis. Mol Clin Oncol 7, 76-80, 2017.

Mejia-Montilla J, Reyna-Villasmil E, Domínguez-Brito L, Naranjo-Rodríguez C, Noriega-Verdugoc D, Padilla-Samaniego M, Vargas-Olalla V. Suplementacion de acidos grasos omega-3 y adiponectina plasmatica en mujeres con sindrome de ovarios poliquisticos. Endocrinol Diabetes Nutr 65, 192-199, 2018.

Mirmasoumi G, Fazilati M, Foroozanfard F, Vahedpoor Z, Mahmoodi S, Taghizadeh M, Esfeh NK, Mohseni M, Karbassizadeh $\mathrm{H}$, Asemi Z. The Effects of flaxseed oil omega-3 fatty acids supplementation on metabolic status of patients with polycystic ovary syndrome: A randomized, double-blind, placebo-controlled trial. Exp Clin Endocrinol Diab 126, 222-228, 2018.

Mohammad-Alizadeh-Charandabi S, Mirghafourvand M, Froghy L, Javadzadeh Y, Razmaraii N. The effect of multivitamin supplements on continuation rate and side effects of combined oral contraceptives: A randomised controlled trial. Eur J Contracep Reprod Health Care 20, 361-371, 2015.

Mohammadi E, Rafraf M. Benefits of omega-3 fatty acids supplementation on serum paraoxonase 1 activity and lipids ratios in polycystic ovary syndrome. Health Promot Perspect 2, 197-204, 2012.

Mohammadi E, Rafraf M, Farzadi L, Asghari-Jafarabadi M, Sabour S. Effects of omega-3 fatty acids supplementation on serum adiponectin levels and some metabolic risk factors in women with polycystic ovary syndrome. APJCN 21, 511-518, 2012.

Moran LJ, Pasquali R, Teede HJ, Hoeger KM, Norman RJ. Treatment of obesity in polycystic ovary syndrome: a position statement of the Androgen Excess and Polycystic Ovary Syndrome Society. Fertil Steril 92, 1966-1982, 2009.

Rafraf M, Mohammadi E, Asghari-Jafarabadi M, Farzadi L. Omega-3 fatty acids improve glucose metabolism without effects on obesity values and serum visfatin levels in women with polycystic ovary syndrome. JACN 31, 361-368, 2012.

Nadjarzadeh A, Dehghani-Firouzabadi R, Daneshbodi H, Lotfi MH, Vaziri N, Mozaffari-Khosravi H. Effect of Omega-3 supplementation on Visfatin, adiponectin, and anthropometric indices in women with polycystic ovarian syndrome. J Reprod Infertil 16, 212-220, 2015. 
Najafi H, Changizi-ashtiyani S, Najafi M. Antioxidant activity of omega-3 derivatives and their elivery via nanocages and nanocones: DFT and experimental in vivo investigation. J Mol Model 23, 326, 2017.

Nybacka A, Carlstrom K, Stahle A, Nyren S, Hellstrom PM, Hirschberg AL. Randomized comparison of the influence of dietary management and/or physical exercise on ovarian function and metabolic parameters in overweight women with polycystic ovary syndrome. Fertil Steril 96, 1508-1513, 2011.

Oner G, Muderris II. Efficacy of omega-3 in the treatment of polycystic ovary syndrome. J Obstetr Gynecol 33, 289-291, 2013.

Phelan N, O'Connor A, Tun TK, Correia N, Boran G, Roche HM, Gibney J. Hormonal and metabolic effects of polyunsaturated fatty acids in young women with polycystic ovary syndrome: results from a cross-sectional analysis and a randomized, placebo-controlled, crossover trial. JACN 93, 652-662, 2011.

Palmery M, Saraceno A, Vaiarell A, Carlomagno G. Oral contraceptives and changes in nutritional requirements. Eur Rev Med Pharmacol Sci 17, 1804-1813, 2013.

Rafraf M, Mohammadi E, Asghari-Jafarabadi M, Farzadi L. Omega-3 fatty acids improve glucose metabolism without effects on obesity values and serum visfatin levels in women with polycystic ovary syndrome. JACN 31, 361-368, 2012.

Rahmani E, Samimi M, Ebrahimi FA, Foroozanfard F, Ahmadi S, Rahimi M, Jamilian M, Aghadavod E, Bahmani F, Taghizadeh M, Memarzadeh MR, Asemi Z. The effects of omega-3 fatty acids and vitamin E co-supplementation on gene expression of lipoprotein (a) and oxidized low-density lipoprotein, lipid profiles and biomarkers of oxidative stress in patients with polycystic ovary syndrome. Mol Cell Endocrinol 439, 247-255, 2016.

Rahmani E, Jamilian M, Dadpour B, Nezami Z, Vahedpoor Z, Mahmoodi S Aghadavod E, Taghizadeh M, Beiki Hassan A and Asemi Z. The effects of fish oil on gene expression in patients with polycystic ovary syndrome. EJCI 48, e12893, 2018.

Sadeghi A, Djafarian K, Mohammadi H, Shab-Bidar S. Effect of omega-3 fatty acids supplementation on insulin resistance in women with polycystic ovary syndrome: Meta-analysis of randomized controlled trials. Diabetes Metab Syndr: Clin Res Rev 11, 157-162, 2017.

Sadeghi F, Alavi-Naeini A, Mardanian F, Ghazvini MR, Mahaki B. Omega-3 and vitamin E co-supplementation can improve antioxidant markers in obese/overweight women with polycystic ovary syndrome. Int J Vit Nutr Res 90, 477-483, 2020.

Silva RC, Pardini DP, Kater CE. Polycystic ovary syndrome, metabolic syndrome, cardiovascular risk and the role of insulin sensitizing agents. Braz Arq Endocrinol Metabol 50, 281- 290, 2006.

Silva DEA. Prevalence of metabolic disorders in polycystic ovarian syndrome. RBONE-Rev Bras Obes Nutr Emagr 7, 2, 2013. (Abstract)

Stegeman BH, Bastos M, Rosendaal FR, Vlieg AVH, Helmerhorst FM, Stijnen T, Dekkers OM. Different combined oral contraceptives and the risk of venous thrombosis: systematic review and network meta-analysis. BMJ 347, f5298, 2013.

Talari HR, Poladchang S, Hamidian Y, Samimi M, Gilasi HR, Ebrahimi FA, Asemi Z. The effects of omega-3 and vitamin $\mathrm{E}$ co-supplementation on carotid intima-media thickness and inflammatory factors in patients with polycystic ovary syndrome. OMJ 33, 473-479, 2018.

Thota RN, Ferguson JJA, Abbott KA, Dias CB, Garg ML. Science behind the cardio-metabolic benefits of omega-3 polyunsaturated fatty acids: Biochemical effects vs. clinical outcomes. Food Funct 9, 3576-3596, 2018.

Unluhizarci K, Kaltsas G, Kelestimur F. Non polycystic ovary syndrome-related endocrine disorders associated with hirsutism. Eur J Clin Invest 42, 86-94, 2012.

Vargas ML, Almario RU, Buchan W, Kim K, Karakas SE. Metabolic and endocrine effects of long-chain versus essential omega-3 polyunsaturated fatty acids in polycystic ovary syndrome. Metabolism 60, 1711-1718, 2011.

Yang K, Zeng L, Bao T, Ge J. Effectiveness of Omega-3 fatty acid for polycystic ovary syndrome: a systematic review and meta-analysis. Repr Biol Endocrinol 16, 27, 2018.

Yao K, Bian C, Zhao X. Association of polycystic ovary syndrome with metabolic syndrome and gestational diabetes: Aggravated complication of pregnancy. Exp Ther Med 14, 1271-1276, 2017. 